\title{
The maternal autopsy
}

\author{
DI RUSHTON, IMP DAWSON \\ From the Department of Pathology, Birmingham Maternity Hospital, Birmingham, and the Department of \\ Pathology, University Hospital, Nottingham
}

SUMmaRy Careful study of reports prepared for the Confidential Enquiries into Maternal Deaths in England and Wales has made it clear that many maternal autopsy reports are not as informative as they might be. This is, in part at least, because no pathologist who does not work in a maternity unit can expect to see more than a handful of such deaths in a working lifetime. This paper describes briefly the particular features to look for at autopsy, stresses the importance of taking adequate material for histology and discusses some of the more significant histological findings, both of conditions which cause death and of those commonly associated with it.

Many pathologists may not be familiar with the Reports on Confidential Enquiries into Maternal Deaths in England and Wales. These have been published triennially by HM Stationery Office since the first report covering the years 1952/54 and are based on case notes and autopsy reports from individual patients.

The most recent published report covering the years $1973 / 75^{1}$ was critical of some of the autopsy reports available. Thus the Chief Medical Officer in his preface wrote:

"The importance of accurate diagnosis of the cause of death becomes greater as the number of deaths diminish. Post-mortem examinations were carried out in 344 out of the 390 deaths investigated, but the authors and the regional assessors have all expressed concern that the reports available were not all as helpful as they might have been. The assessors need full details of the post-mortem and all histological examinations. More discussion between the clinicians and the pathologists as to the cause of death at the time of examination might help to elucidate some of the problems."

Within the report itself, under the heading "Post-mortem Examinations" the Central Assessors commented as follows:

"In many cases lack of detail in the post-mortem reports, and in other cases apparently inadequate post-mortem examinations, made it difficult to assess the cause of death with accuracy. For example, in 6 out of 36 autopsies the source of pulmonary embolism was not even looked for, and microscopic

Accepted for publication 11 May 1982 examination of the lungs was omitted in several cases of suspected amniotic fluid embolism.

It is clearly to be regretted that the post-mortem examination, which can contribute so much to the ultimate solution of the cause of death, is so incompletely performed in many cases. Pathologists surely have a responsibility to support their clinical colleagues by undertaking searching autopsies, which must involve the carrying out of all the appropriate measurements, both macroscopic and microscopic, to confirm or refute the apparent clinical cause of death."

As a result of these criticisms the consultant advisor in Histopathology to the DHSS (IMPD) was invited to join the Central Assessors in Obstetrics and Anaesthetics in the preparation of the Report for $1976 / 78$. Subsequently, the CMO decided, in consultation with The Royal Colleges of Pathologists and Obstetricians and Gynaecologists to set up a panel of Regional Assessors in Pathology similar to those already established in Obstetrics and Anaesthetics. This panel first met in June 1981 with the Central Assessors and, after discussion, proposed that in each region there should be a small panel of histopathologists on whom colleagues might call for advice on the special problems in autopsies on maternal deaths and upon whom HM Coroner might feel he could depend for specialised assistance. These have now been set up.

\section{Causes of maternal death}

Deaths investigated in the Confidential Enquiry are divided into true maternal deaths which are directly 
Table 1 Deaths due to or associated with pregnancy and childbirth 1973-1975

\begin{tabular}{llr}
\hline & & Cases \\
\hline I & Infections & 4 \\
II & Neoplasms & 29 \\
III & Endocrine disorders & 10 \\
IV & Blood diseases & 3 \\
V & Mental disorders & 1 \\
VI & Central nervous system diseases & 10 \\
VII & Circulatory diseases & 38 \\
VIII & Respiratory diseases & 9 \\
IX & Digestive diseases & 8 \\
X & Genitourinary diseases & 6 \\
XI & Complications of pregnancy, childbirth & 232 \\
& and puerperium & 1 \\
XIII & Musculoskeletal disorders & 10 \\
XIV & Congenital anomalies & 29 \\
EXVII & Accidents, poisoning and violence & \\
\hline
\end{tabular}

Table 2 Complications of pregnancy, childbirth and the puerperium 1973-75

\begin{tabular}{llr}
\hline ICD No & Complication & Cases \\
\hline 631 & Ectopic pregnancy & 21 \\
632 & Haemorrhage of pregnancy & 3 \\
634 & Other complications of pregnancy & 20 \\
637 & Pre-eclampsia, eclampsia and & 39 \\
& toxaemia unspecified & 2 \\
638 & Hyperemesis & 5 \\
639 & Other toxaemia of pregnancy and & 13 \\
& the puerperium & 1 \\
640 & Abortion induced for medical indications & 10 \\
641 & Abortion induced for other legal indications & 5 \\
642 & Abortion induced for other reasons & \\
643 & Spontaneous abortion & 8 \\
& & 4 \\
Delivery & complicated by: & 8 \\
651 & Placenta praevia or antepartum haemorrhage & \\
652 & Retained placenta & 6 \\
653 & Other postpartum haemorrhage & 6 \\
654 & Abnormality of bony pelvis & 8 \\
655 & Fetopelvic disproportion & 4 \\
656 & Malpresentation of the fetus & 10 \\
657 & Prolonged labour of other origin & 1 \\
659 & Rupture of uterus & 5 \\
660 & Other obstetrical trauma & 21 \\
661 & Other complications & 1 \\
670 & Sepsis of childbirth and the puerperium & \\
673 & Puerperal pulmonary embolism & \\
675 & Puerperal blood dyscrasias & \\
\hline & & \\
& &
\end{tabular}

attributable to the pregnancy and associated maternal deaths (see Tables 1 and 2). The associated deaths are subdivided into indirect deaths in which the disease causing death and the pregnancy are interrelated and fortuitous deaths in which the cause of death is in no way related to pregnancy. Maternal deaths are defined by the International FIGO (Federation Internationale de Gynecologie et Obstetrique) classification as deaths occurring during pregnancy or within six weeks of delivery: the Confidential Enquiry extends the postnatal period to a year after delivery and lays particular emphasis on the complications resulting from therapeutic regimens and anaesthesia. Such deaths may present particular difficulties to the pathologist since they generally occur in hospital patients who, following the acute catastrophe, may be maintained on a life support system for a considerable time. When death occurs, the original pathology has often resolved or altered and new factors may have been introduced.

The number of maternal deaths directly due to obstetric causes continues to decline. The most recent published report covering the years 1973/75 considered 390 cases (235 direct, 155 associated) while the as yet, unpublished report for $1976 / 78$ will review 427 deaths (227 direct, 200 associated) occurring over three years. As a result individual pathologists will rarely encounter a maternal death. Indeed if maternal deaths were distributed evenly throughout all Regions, which they are not, there would be only 9-10 cases per annum per Region. The majority of these deaths (194/227 direct, 133/ 200 associated) are reported to HM Coroner and as with all autopsies performed for a Coroner the most important consideration then becomes the determination of the cause of death. In such circumstances much additional pathological information which is desirable may not be looked for or may be inadequately recorded. Since such deaths are among the most tragic any pathologist will encounter, it ie clearly in his or her remit to examine them as fully as possible rather than being satisfied with simplof establishing the cause of death.

The purpose of this paper is not to dictate how such autopsies should be performed but to indicate what particular studies might be valuable as a routine to increase our knowledge and understanding of the complications of both normal and abnormal pregnancies, delivery and the puerperium. We have also described briefly the macroscopic and microscopic appearances of particular conditions which occur in or closely associated with pregnancy, such as fatty change in late pregnancy and "shock lung", since some pathologists may be unfamiliar with them. We have not attempted to be encylopaedic nor to describe conditions such as venous sinus thrombosis which occur in association with pregnancy but with which pathologists will already be familiar.

\section{Clinical details}

It is of paramount importance that a full history is obtained before the autopsy is begun. There are two sources and both should be used. The current case notes should be studied by the pathologist; they record facts as they occurred without hindsight. Some pathologists may not have the expertise to interpret fetal heart rate traces, ultrasound pictures 
and biochemical data, but these must not be ignored because they cannot be interpreted and the advice of a clinical colleague should be obtained if necessary.

When relevant the anaesthetist should be invited to attend the autopsy: this is not usually a problem when the autopsy is done in the hospital concerned, since any obstetrician, anaesthetist or physician involved in a maternal death is normally only too anxious to be present, but it does raise difficulties in some coroner's cases performed in a public mortuary at an inconvenient time and at some distance from the maternity unit. It provides an important opportunity for questioning and discussion though one must remember that a verbal summary at autopsy is likely to be coloured by the ultimate outcome and may not always coincide with what was written in the notes at the time.

In a number of autopsies the combined clinical and pathological data still may not provide an adequate explanation of death and there may be dispute as to precisely what happened-for example, evidence of suspected gastric aspiration may have disappeared if life support systems were temporarily successful. Technical difficulties with anaesthesia may result in cardiac arrest without demonstrable trauma in the upper respiratory tract and autopsy findings in haemorrhage and afibrinogenaemia can be minimal. Iatrogenic pulmonary oedema can follow drugs used to suppress premature labour, in particular the combination of ritodrine and betamethasone. ${ }^{2}$ Water intoxication can follow electrolyte-free infusions of $5 \%$ or $10 \%$ glucose with or without syntocinon. ${ }^{3}$ The resulting hyponatraemia is demonstrable at autopsy by analysis of vitreous humour or of cerebrospinal fluid. ${ }^{4}$ In patients with previously impaired cardiac function the haemodynamic changes which occur normally in the immediate postpartum period can precipitate fatal cardiac failure..$^{s}$

Care should always be taken to determine the nature and dosage of all drugs given during the management of labour, including all anaesthetic and analgaesic agents. Recent evidence suggests that the use of ergometrine for the stimulation of postpartum uterine contraction can lead to bronchospasm in asthmatics. ${ }^{6}$

\section{The autopsy-macroscopic appearances}

An autopsy on a maternal death does not require extensive modification of good standard technique for the external description or the removal of organs, provided that one remembers that certain of the lethal complications of pregnancy are either unique (amniotic fluid embolism, eclampsia) or unusual in non-maternal autopsies (air embolism, pituitary necrosis) so that it may be necessary to use techniques which positively identify their presence or absence. The absence of certain specific abnormalities often needs to be stated categorically. In a proportion of cases there will be a fetus or neonate and a placenta to examine. ${ }^{7-9}$. If possible the pathologist performing the maternal autopsy should also examine and report on this material: at least he should have access to it or to reports of any examinations made on it.

Any woman of child-bearing age may be pregnant at the time of death from whatever cause. On occasion an unsuspected pregnancy or its complications may contribute to an unexpected death. In many deaths attributed to and in many associated with pregnancy the underlying pathology will be suggested on clinical grounds and the appropriate techniques and investigations can be planned. There is $a$ certain amount of mandatory material to be taken into foxative in block form in every case. Subsequent histology may or may not be necessary but the blocks on which this depends must always be available for further study (see below). It is also wise in every case to take samples of heart blood, gastric and intestinal content and urine at the start of an autopsy: these can be discarded later if they are not needed.

Further specific problems are discussed system by system below.

\section{DETECTION OF AIR EMBOLISM}

Air embolism should be suspected in any death which occurs suddenly in abortion or labour; it follows the introduction of a large volume of air $(>150$ $\mathrm{ml}$ ) into the systemic venous circulation which is frothed up by the action of the right ventricle and mechanically blocks the pulmonary trunk. Coronary air embolism associated with rupture of lung veins is not a complication of pregnancy. Open the body by a midline incision from sternal notch to pubic symphysis but do not incise the neck since it is easy to introduce air into severed veins. Next, open the peritoneal cavity and examine the inferior vena cava for the presence in it of frothy blood or air bubbles. If the clinical history suggests it, exclude the presence of pneumothorax and open the chest, again taking care not to cut major veins; incise the pericardium. Examine the superior vena cava and right atrium externally for bloodstained froth or air; fill the pericardial cavity with water and incise right atrium and ventricle beneath the water, observing any bubbles which escape. Delay between death and autopsy with post-mortem growth of gas-producing organisms can mimic air embolism, but similar gas bubbles are usually present also in the left heart, ascending aorta and major branches. 
Collecting blood samples

Before opening the heart further collect a sample of blood from the unopened left ventricle into a sterile container using a large $(50 \mathrm{ml})$ syringe and a wide bore needle. This can be discarded later if not required.

\section{DETECTION OF AMNIOTIC FLUID EMBOLISM}

Remove heart and lungs together without opening the heart further. Carefully incise the pulmonary trunk and open up the right and left pulmonary arteries and their main branches. Look carefully for thrombotic and amniotic emboli. The latter are only rarely visible with the naked eye as lanugo hairs, vernix or meconium and appropriate blocks for histology must always be taken (see below).

An attempt should also be made to identify the portal of entry of any embolus. This necessitates careful examination of the placenta and uterus. It is a prerequisite of amniotic fluid embolism for the amnion to be torn. The tear may be in the amnion enclosing the fore or hind waters so that clinically intact membranes do not exclude the diagnosis. Lethal amniotic fluid embolism is most commonly associated with relatively small tears in the uterus, cervix or vagina which have not totally disrupted the wall (Fig. 1). This is perhaps to be expected since a complete tear will allow the majority of the amniotic fluid to escape into the peritoneal cavity or to the vagina. It is therefore essential that the uterus, cervix and vagina be examined very minutely and in the authors' experience this is best done after fixation of the opened uterus.

\section{CARDIOVASCULAR SYSTEM}

Always weigh the heart. Routinely take blocks through the midregion of left and right ventricles to exclude cardiomyopathy. Look carefully for dissecting aneurysms of aorta or coronary arteries ${ }^{10}$ or local aneurysm of mesenteric, splenic, hepatic, uterine or ovarian arteries. ${ }^{1112}$

\section{RESPIRATORY SYSTEM}

Before opening the chest look for evidence of pneumothorax. Carefully examine upper respiratory tract for evidence of trauma and aspiration of gastric contents especially in patients who have died under or shortly after anaesthesia. Look for macroscopic evidence suggesting amniotic fluid embolism (see above), shock lung and respirator (ventilator) lung if appropriate. In shock lung, which can follow severe haemorrage or trauma, be associated with Gramnegative septicaemia ${ }^{1314}$ and can be made worse by therapy including oxygen administration and uncontrolled hydration, the lungs usually drip bloodstained fuid on section and show patchy purplish solid zones which are predominantly basal. In respirator (ventilator) lung ${ }^{15}$ which follows the use of prolonged positive pressure ventilation (Fig. 2) two abnormalities should be looked for. If, as is probable, a tracheostomy has been performed and a cuffed tracheostomy tube inserted there may be tracheal ulceration; alternatively the use of an endotracheal tube can produce laryngeal damage. Positive pressure respiration is usually associated with oxygen administration and carries the risks of oxygen damage leading to intra-alveolar haemorrhage. Macroscopic appearances are similar to those of shock lung, and the histological appearances are mentioned below. Take blocks for histology from all lobes of both lungs. Bacterial, and where practical viral studies should be made on all clinically unexpected or unexplained deaths and on all deaths under anaesthetic.

\section{ALIMENTARY SYSTEM}

Look for trauma in the upper oesophagus which may be associated with problems of intubation. Note any gastric or small bowel dilatation or ileus, and if surgery or abortion have been carried out, look carefully for perforations: retain stomach and small bowel content if poisoning (suicide, drug reaction) is suspected. Examine the liver carefully and record its weight; look for evidence of fatty change, necrosis or haemorrhage, always take blocks for histology, and preserve a piece frozen and unfixed in case toxocological examination is needed. Acute fatty change in pregnancy ${ }^{1617}$ is a specific disorder which should always be looked for histologically (see below).

\section{URINARY SYSTEM}

Look carefully for trauma to bladder and urethra. Weigh both kidneys and take blocks from each for histology. A sample of urine should always be taken.

\section{GENITAL ORGANS}

Prior to removal, examine the vulva, vagina, cervix and uterus carefully for evidence of trauma and tears. Then remove the genitalia intact en bloc; it is often necessary to split the symphysis pubis to do this. Open the vagina, cervix and uterus anteriorly. Look for previous scars and search carefully for internal tears. Note any placental remnants and any abnormal amount of blood clot. Is there any uterine inversion? Exclude tubal pregnancy. If possible preserve the entire genital tract in fixative; if not take appropriate tissue blocks (see below).

\section{CENTRAL NERVOUS SYSTEM}

The pathologist should remove the skull vault and brain himself. Look for venous sinus thrombosis 


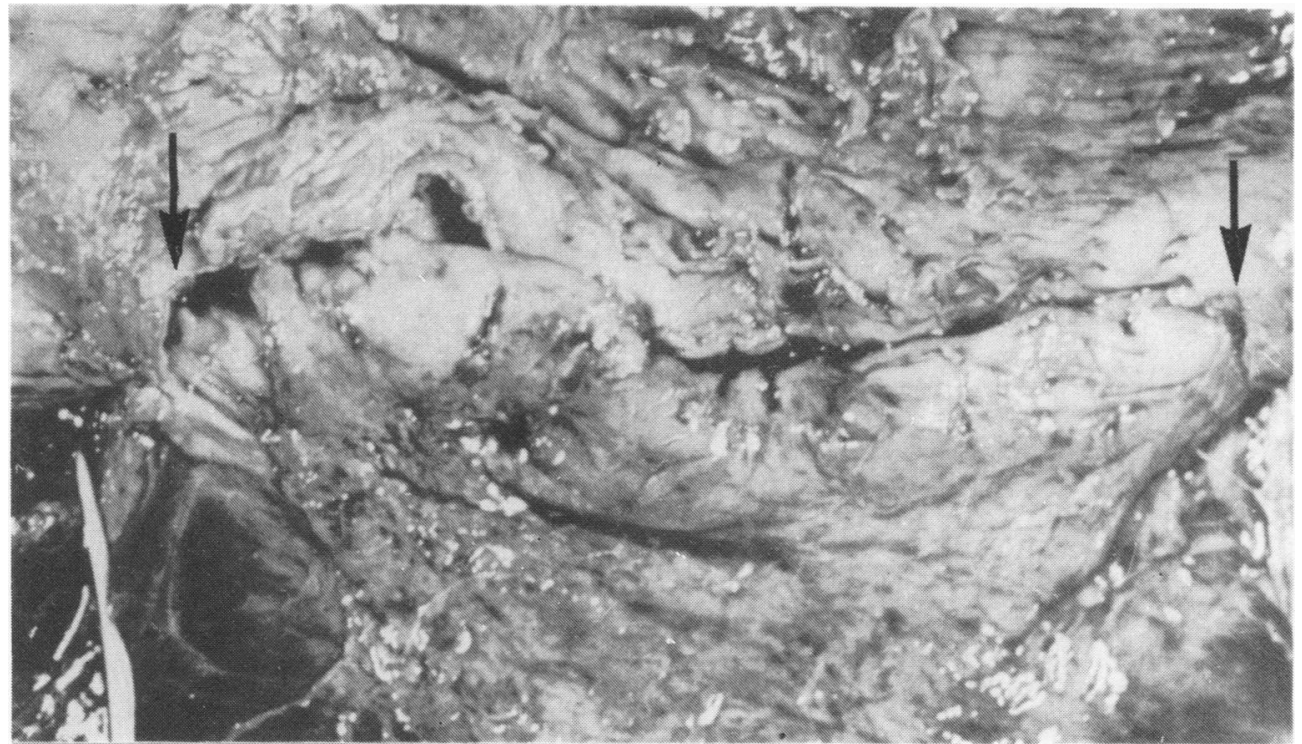

Fig. 1 Lower uterine segment with post-mortem caesarean section wound (between arrows). A probe enters a torn vein, the site of an amniotic fuid embolism $\times 1.5$

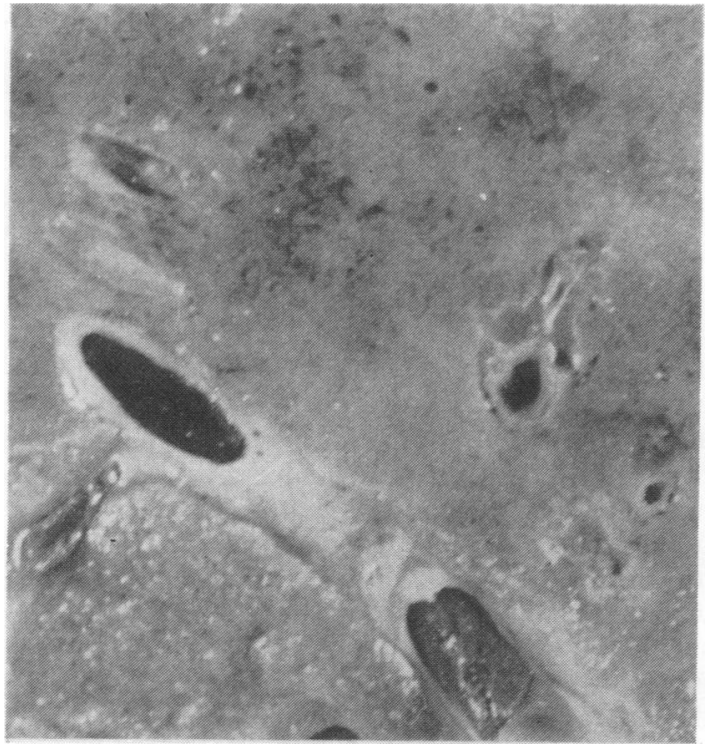

Fig. 2 Ventilator lung $\rightarrow$ solid pale airless lung. An embolus is present in a small pulmonary artery $\times 2$

particularly in postpartum and puerperal deaths and where there is any history suggesting an epileptiform fit. Fix brain suspended prior to slicing if possible. Cerebal haemorrhage in non-hypertensive maternal deaths may be due to metastatic chorion carcinoma so always take blocks from all brains in which haemorrhage has been identified. Remove and preserve pituitary in fixative routinely. If spinal or epidural anaesthesia was used, examine spinal canal and cord for possible trauma or infection.

\section{OTHER TISSUES}

Always weigh the spleen and look for tears in it. If the history warrants examine the cervical spine (difficulty in intubation), pelvis (bony abnormality complicating delivery) and costochondral junctions (rickets especially in Asian women).$^{18}$ If these complications are suspected and $x$-ray facilities are available, appropriate $x$-ray should be performed. Examine the bone marrrow in sternum and in a long bone. Look carefully at the adrenals to exclude pleochromocytoma. ${ }^{19}$

\section{Blocks for histology}

In the experience of the Central and Regional Obstetric Assessors the single most significant defect in the maternal autopsies has been the lack of available histology. There are certain blocks which should be taken routinely into fixative at any maternal autopsy and subsequently processed at least to paraffin. Even if the pathologist who did the autopsy does not wish to examine them they are then available and may provide vital evidence in the explanation of a train of events which led to death. In our view, the following should always be taken for histology at any maternal autopsy: 


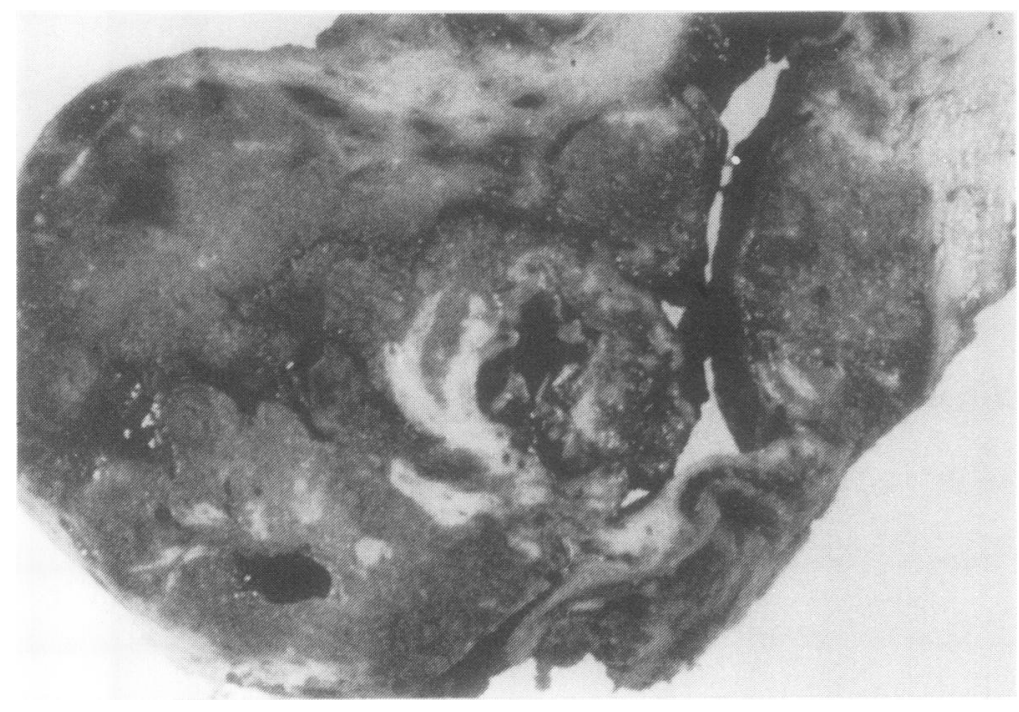

Fig. 3 Grade IV placenta praevia invading the cervix resulting in placenta percreta $\times 1.5$

(i) blocks from any abnormal tissue found at autopsy-for example, an inflamed trachea or the edge of a cerebral haemorrhage.

(ii) blocks from the following sites even if no abnormality was visible: pituitary; right and left ventricles; right upper middle and lower lung lobes; left upper and lower lung lobes; liver; right and left kidneys; right and left ovaries; right and left fallopian tubes; placental site of uterus; cervix.

\section{Examination of placenta and products of conception}

It is clearly impracticable to detail techniques for examination of the placenta. In many maternal deaths the placenta will have been delivered and disposed of without, at most, more than a superficial examination. In those cases where it is available either in situ or delivered it is recommended that it is fully examined, particular emphasis being laid on lesions which may be related to the cause of death of the mother. These include:

(i) morbidly adherent placenta

(ii) amniotic fluid embolism (vide supra)

(iii) retroplacental haemorrhage

(iv) uteroplacental ischaemia ${ }^{20}$

(v) embryonic or fetal death prior to delivery ${ }^{9}$

(vi) retained products of conception

(vii) infection and criminal abortion"

(viii) trophoblastic disease. ${ }^{21}$

Histological preparations should be made of all placentae from maternal deaths to include cord, membranes, central and marginal placental tissue and macroscopically identifiable lesions.
ABNORMAL PLACENTATION

Abnormal placentation is an important factor in a significant proportion of deaths due to haemor 8 rhage. There are two major patterns of abnormalityo

(i) abnormalities of site typified by ectopic prege nancy and placenta praevia (Fig. 3)

(ii) abnormalities of attachment typified by the morbidly adherent placenta (placenta accreta, increta and percreta) (Fig. 4). Placenta praevia may be complicated by adherence particularly if the placenta is attached over a previous lower segment caesarean section scar.

The diagnosis of placenta praevia is almost invariably made clinically though occasionally fatal antepartum haemorrhage occurring in the absence of medical attention may require the pathologist to make the diagnosis. It is therefore particularly important that the uterus be removed with the vagina and the cervix so that it may be examined intact when the pregnancy is undelivered. Since it is lower segment implantation of the placenta which is particularly hazardous the uterus is best opened from the fundus so that a placenta completely covering the lower segment or internal os of the cervix can be clearly demonstrated in situ. Having opened the uterus to display the placenta, the fetus may be removed and the uterus and placenta fixed prior to slicing the specimen. In postpartum cases examination of the vessels in the lower segment and cervix may reveal the characteristic adaptive changes described above in addition to remnants of villi or trophoblast. An extremely rare form of placenta praevia is that associated with placenta mem- 


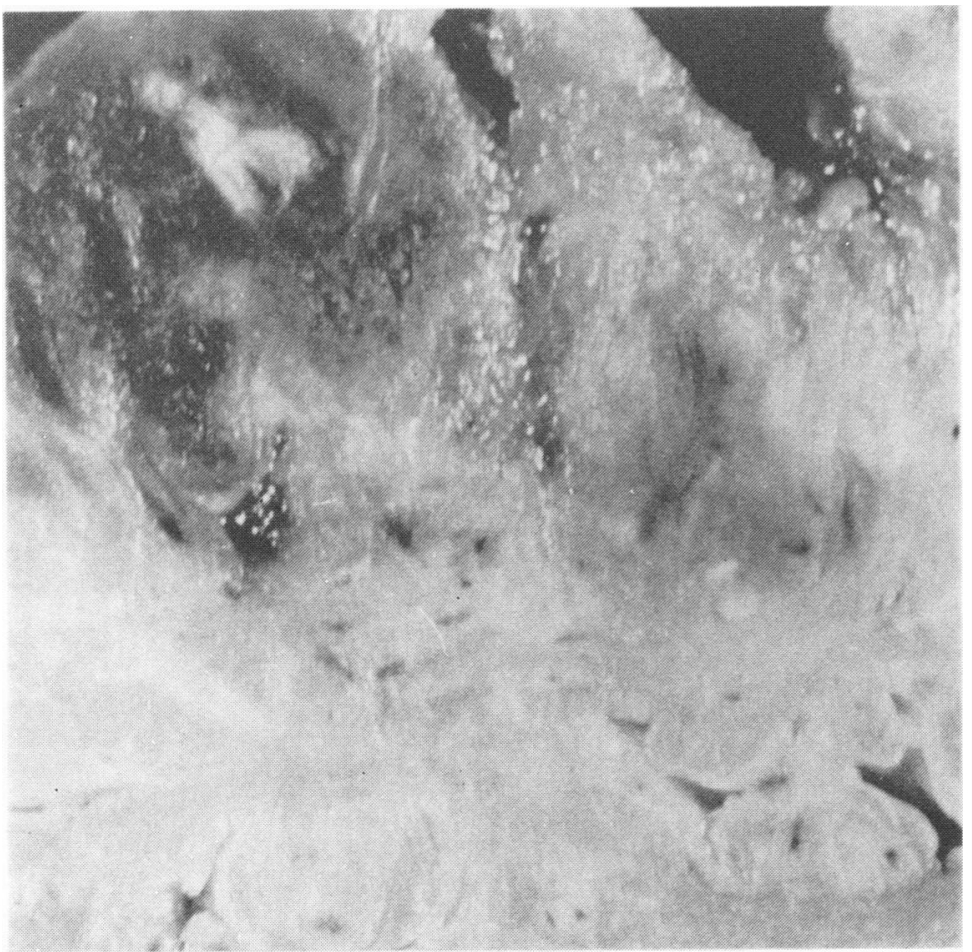

Fig. 4 Placenta accreta-nodules of placental tissue inderdigitate with the myometrium containing numerous vessels $\times 2.5$

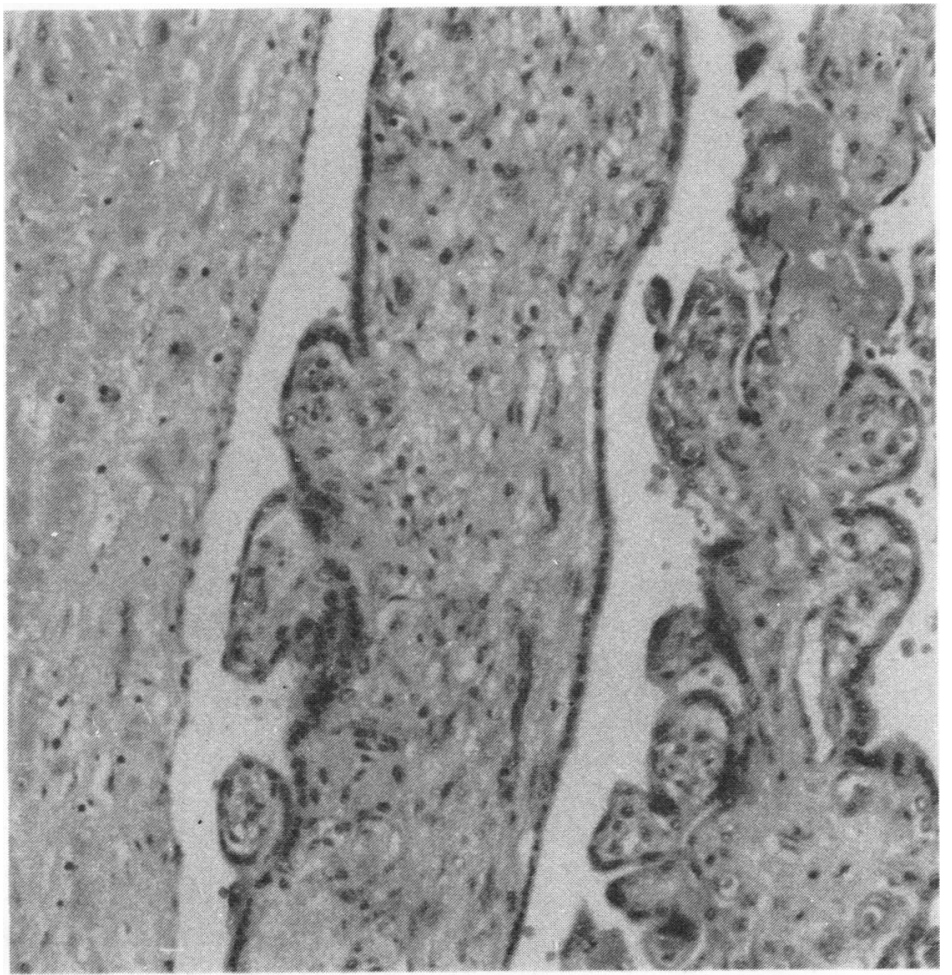

Fig. 5 Placenta accreta-placental villi abut directly on the myometrium. There is no intervening decidua. Haematoxylin and $\operatorname{eosin} \times 192$ 


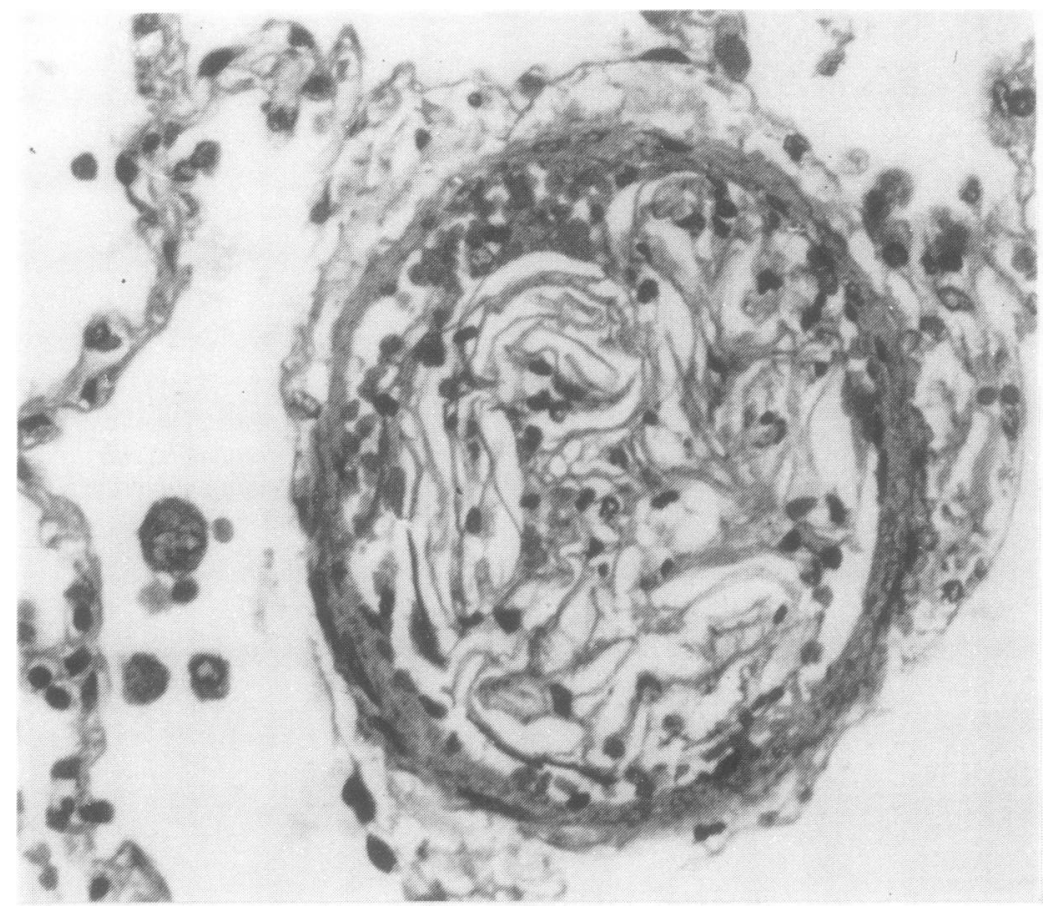

Fig. 6 Amniotic fuid embolus containing numerous squames in pulmonary artery. Attwood's stain $\times 395$

branacea where the entire chorionic plate is covered by villous tissue. It may be associated with a morbidly adherent placenta.

Morbidly adherent placenta has been reviewed by Fox $^{20}$ who culled 622 cases from the literature. It occurs in between 1 in 540 and 1 in 93000 deliveries. The aetiology is unknown but a deficiency in the endometrium at the site of implantation appears probable. About one third of cases are associated with placenta praevia and in over half there is a history of previous uterine surgery (dilatation and curettage, caesarean section). There is frequently clinical evidence of difficulty in delivering the placenta which is often incomplete. Careful examination of the postpartum uterus is essential and all raised lesions as well as those clearly related to retained products must be examined histologically. The characteristic finding is the direct apposition of villous and trophoblastic elements to the myometrium without the normal intervening layer of decidua (Fig. 5). In the most severe form, placenta percreta, the villi may penetrate the full thickness of the cervix or myometrium and invade adjacent structures such as the bladder. In all patients in whom a definite source of haemorrhage is not demonstrated macroscopically multiple blocks of the placental bed should be examined for abnormally adherent placental tissue.

\section{Specialised histopathology in pregnancy}

A number of patients die, usually shortly after delivery, with a clinical syndrome in which any of the components shock, hypotension, a generalised bleeding diathesis or non-coagulability of the blood may predominate. Three conditions, which are often interrelated, though separately diagnosable, namely amniotic fluid embolism, disseminated intravascular coagulation (DIC) and Gram-negative septicaemia should all be considered and supportive diagnostic evidence sought.

\section{AMNIOTIC FLUID EMBOLISM}

This subject has been well reviewed. ${ }^{22}$ Macroscopic diagnosis can occasionally be made by identifying meconium, vernix and lanugo hairs in blood from the pulmonary arteries, but histological examination of lungs is always necessary. The histological components of amniotic fluid emboli have been defined as follows: ${ }^{23}$

1 Epithelial squames shed from the fetal skin (Fig. 6)

2 Lanugo hairs-these increase in number with gestation

3 Fatty material-vernix caseosa (Fig.7)

4 Mucin-generally derived from the fetal intestine and associated with passage of meconium 


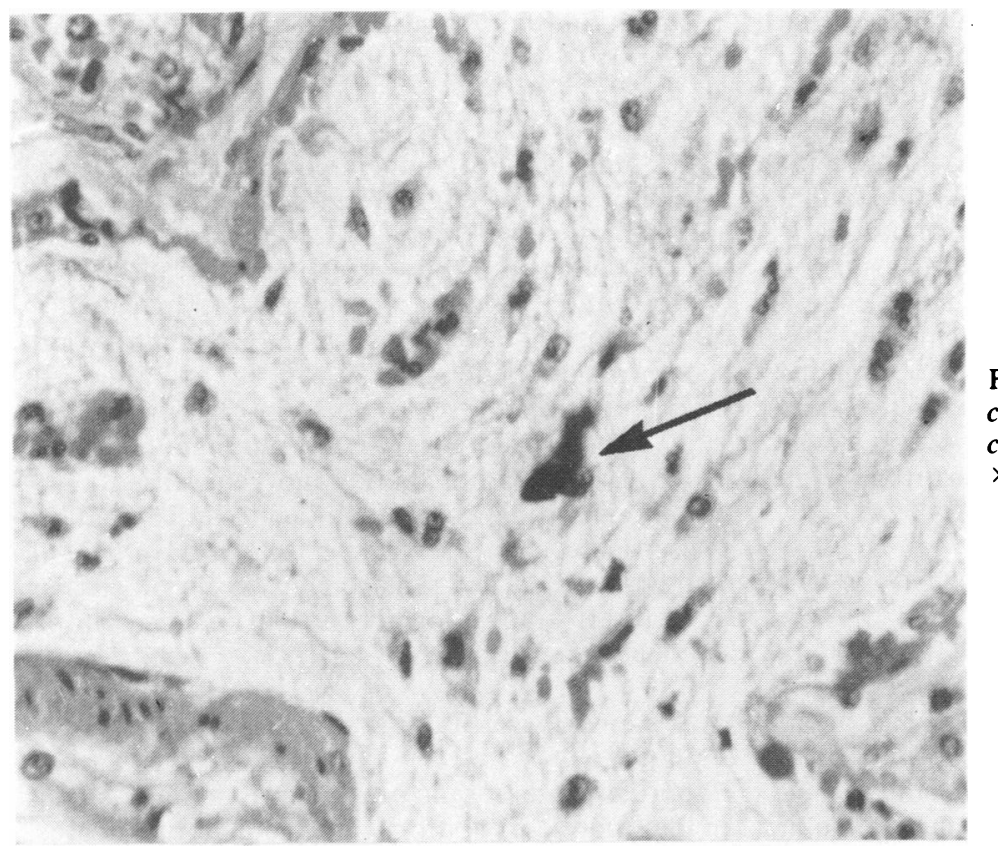

Fig. 7 Amniotic fuid embolus consisting largely of mucin with vernix caseosa (arrowed). Attwood's stain $\times 395$

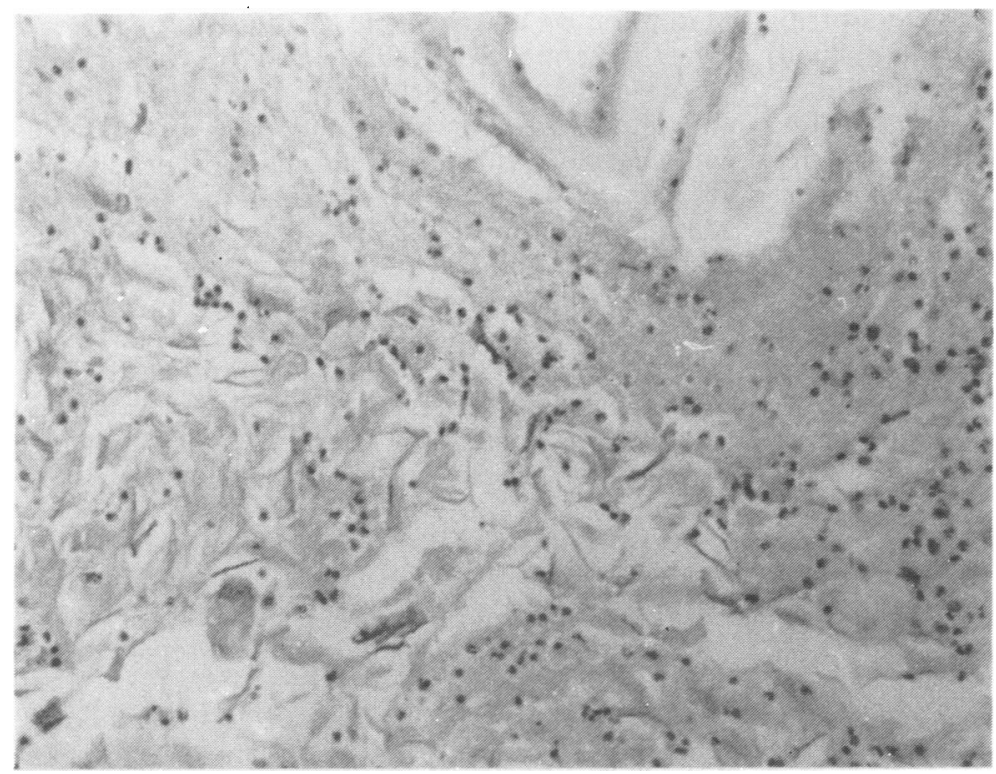

Fig. 8 Squames and polymorphs dissecting the placental membranes. Haematoxylin and eosin $\times 192$

5 Bile pigments derived from the meconium. While all these components can be identified in routine haematoxylin and eosin stained sections they may easily be overlooked. An alcian bluephloxine-tartrazine technique ${ }^{24}$ should be routinely employed on lung sections in all maternal deaths, whether the diagnosis is suspected or not. It is equally applicable to material in uterine vessels.
Microscopy of the tear and adjacent tissues may reveal amniotic components. Similar studies on the placenta may reveal squames and other debris dissecting between the amnion and chorion (Fig. 8) True transplacental embolism is very unusual but the risk may be increased if the transplacental approach to the amniotic sac has been used for diagnostic tests. 
Apart from the confirmation of the diagnosis an attempt should also be made to quantify the size of the embolus since not all amniotic fluid emboli are fatal. Such quantification can only be very approximate as the histologically identifiable components are not uniformly distributed from patient to patient even when the periods of gestation are similar. Fatal embolism is usually readily apparent even on haematoxylin and eosin staining while small emboli require careful searching of sections stained by special techniques. Not all deaths related to amniotic fluid embolism occur as a result of acute pulmonary circulatory failure; a significant proportion result from haemorrhage subsequent to disturbance in coagulation, which is discussed below.

DISSEMINATED INTRAVASCULAR COAGULATION (DIC)

Disseminated intravascular coagulation is a phenomenon common to a number of different disease processes, in which there can be widespread deposition of platelets and altered fibrinogen in small vessels, especially capillaries and small arterioles though these are not always detectable at autopsy. ${ }^{256}$ If the condition is sufficiently severe to consume enough normal coagulation factors or to cause damage to end organs by reducing the blood supply to them, haemorrhagic states, shock phenomena and death can ensue. Maternal deaths associated with DIC are seen particularly in patients with pre-eclampsia, amniotic fluid embolism, Gram-negative septicaemia and incompatible transfusion reactions. ${ }^{25-27}$ It is likely that appropriate haematological studies during life will have established the diagnosis, but there are pointers discoverable at autopsy. Macroscopically there may be evidence of generalised bleeding with purpura and ecchymosis widely distributed. Histologically microthrombi may be seen in small vessels and a Martius Scarlet-Blue (MSB) stain is often helpful; absence of thrombi does not exclude the diagnosis since fibrinolysins are often present in life in this condition.

\section{GRAM-NEGATIVE SEPTICAEMIA}

Infection with Gram-negative organisms produces endothelial damage in small vessels which can lead to shock and also to a DIC syndrome; indeed septicaemia and amniotic embolism are closely linked with DIC and can produce related syndromes. Haemorrhages are usually less common at necropsy, but microthrombi can be found in tissues. ${ }^{14}$

In all deaths where one of the triad of DIC, septicaemia or amniotic embolism are suspected, the other two should always be looked for: postmortem blood culture, adequate search for amniotic emboli in lungs and for microthrombi in tissues are all obligatory.

CHANGES IN SHOCK AND RESPIRATOR LUNG Shock lung, already commented on macroscopically, can be present in DIC, Gram-negative septicaemia and amniotic fluid embolism. "Respirator" lung can follow prolonged positive pressure respiration. Microscopically ${ }^{13-15}$ both are characterised by capillary congestion and alveoli which may contain blood, haemorrhagic oedema fluid or be collapsed. In shock lung microthrombi, platelet aggregates and local accumulation of polymorphs can be found within capillaries but there is no generalised neutrophil emigration as is seen in acute imflammation. These acute changes may be followed by hyaline membrane and later replacement by fibrous tissue. In respirator lung the more usual finding is intraalveolar haemorrhage. ${ }^{15}$

PRE-ECLAMPSIA, ECLAMPSIA AND

HYPERTENSION

Hypertensive disease in pregnancy is common and is an important contributory cause to maternal deaths. Clinically the hypertension may be evident prior to conception or may become manifest during preg-8 nancy. Essential hypertension may be complicated by pre-eclampsia or eclampsia. In either situation the uterine vascular bed often provides valuable pathological evidence confirming the clinical assessment and should be examined in all maternal deaths. Hypertensive diseases may produce widespread lesions in many organs but these are outside the remit of this discussion. The pathology of toxaemia of pregnancy has been fully reviewed. ${ }^{28}$

The normal physiological adaption of uterine blood flow during pregnancy results in profound modifications to the spiral arteries (Fig. 9) supplying the placenta. ${ }^{29}$ These changes may be summarised as follows:

(i) replacement of the endothelium of placental bed spiral arteries within the decidua by endovascular trophoblast

(ii) disruption of the arterial wall and replacement by fibrinoid material

(iii) a second wave of endovascular trophoblastic spread (occurring between 12 and 16 weeks gestation) involving the myometrial segments of the spiral arteries

(iv) replacement of the elastic and muscular components of these segments by fibrinoid material.

The basal arteries are not invaded by trophoblast and retain a normal appearance; the radial and arcuate vessels retain their elastic tissue but undergo hypertrophy.

These changes are modified in patients with both 


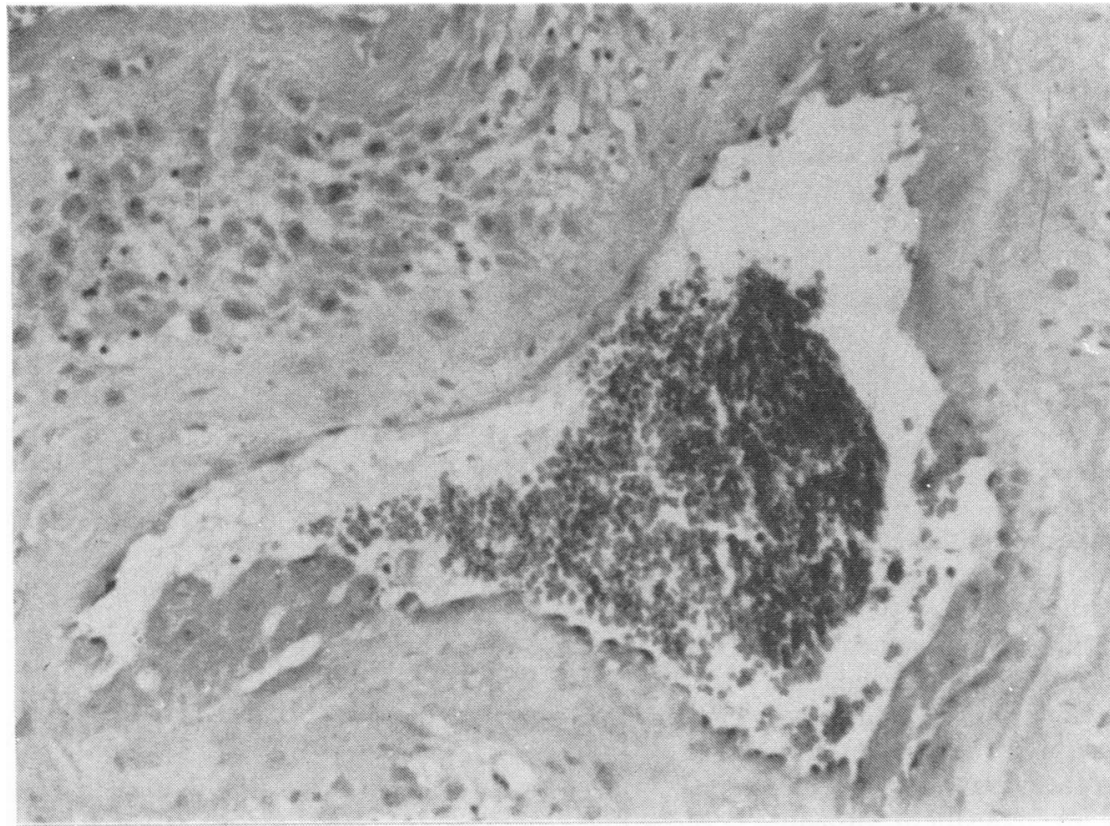

Fig. 9 Spiral artery

showing pregnancy adaption with destruction of the elastica and media and partial replacement of the intima with trophoblast.

Haematoxylin and eosin $\times 192$

essential hypertension and pre-eclamptic toxaemia. In essential hypertension the characteristic lesion is hyperplastic arteriosclerosis notably in the myometrial segments of the spiral arteries. The severity of the lesions in the uterine vessels is frequently much greater than in other organs. In uncomplicated essential hypertension the depth of endovascular trophoblastic invasion and vascular destruction are normal.

In pre-eclamptic toxaemia there are two major abnormalities in the uterine vasculature:

(i) fibrinoid necrosis associated with large numbers of lipophages in the vessel wall, the latter being cuffed by mononuclear cells (Fig.10). Since the lipid in the macrophages derives in part from smooth muscle cells it follows that this lesion may only be found in vessels which have not undergone adaption as described above. Thus it is typically found in the terminations of the spiral arteries in the decidua parietalis, the basal arteries of the decidua basalis and myometrium and in the myometrial segments of the spiral arteries of the placental bed.

(ii) failure of the physiological adaptive changes of the spiral arteries in the placental bed to extend beyond the deciduo-myometrial junction.

The first of these changes is considered to be virtually pathognomonic of pre-eclampsia, ${ }^{30}$ though this has been disputed by others ${ }^{31}$ who have reported similar lesions in "small for dates" pregnancies with

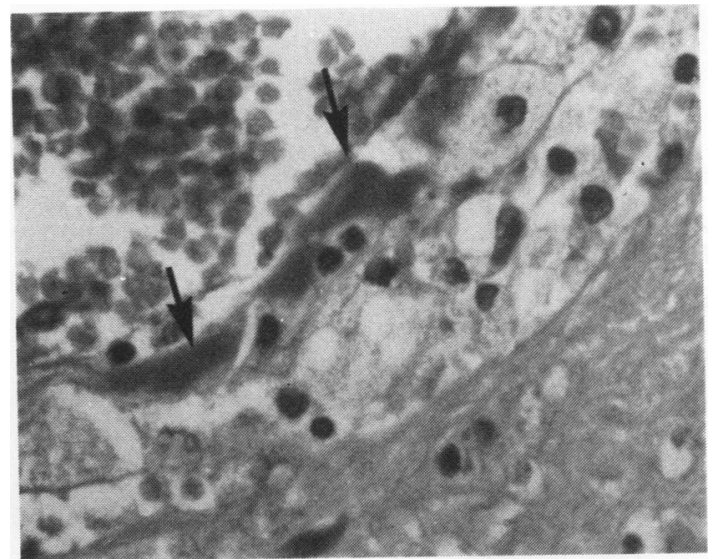

Fig. 10 Lipophages with foamy cytoplasm and deposits of fibrin (arrowed) in decidual vessel of patient with pre-eclamptic toxaemia $($ M.S.B. $\times 395)$.

no evidence of pre-eclampsia. Be that as it may, the examination of the uterine vasculature underlying the placenta should form an integral part of the histological studies performed on the uteri of all maternal deaths. Examination of uterine vessels associated with postpartum haemorrhage frequently reveals that haemorrhage has arisen from arteries which, as the result of physiological adaptation, are unable to constrict after delivery. 
ACUTE FATTY LIVER OF PREGNANCY

Acute fatty liver in late pregnancy can present with abdominal pain and recurrent vomiting without prior warning or jaundice. It has to be distinguished from viral hepatitis and pre-eclampsia. The salient features are the presence of fat in small vacuoles in centrilobular hepatocytes, while a ring of normal hepatocytes remains around portal systems. ${ }^{16} \mathrm{Nec}-$ rosis is not a feature (Figs. 11, 12). There is commonly associated renal failure with tubular necrosis. A similar picture can be produced by prolonged intake of tetracyclines.

\section{Anaesthetic deaths}

These are frequently the most problematic investigations and the role of the pathologist is often confined to looking for evidence of inhalation of stomach contents, damage to larynx or trachea after intubation procedures, determining the presence or absence of respirator lung, and excluding other possible causes of death. The need for accurate and full clinical data is nowhere more important since the morphological evidence of an anaesthetic disaster may be minimal. These patients by their very nature are likely to be placed on life support systems result-

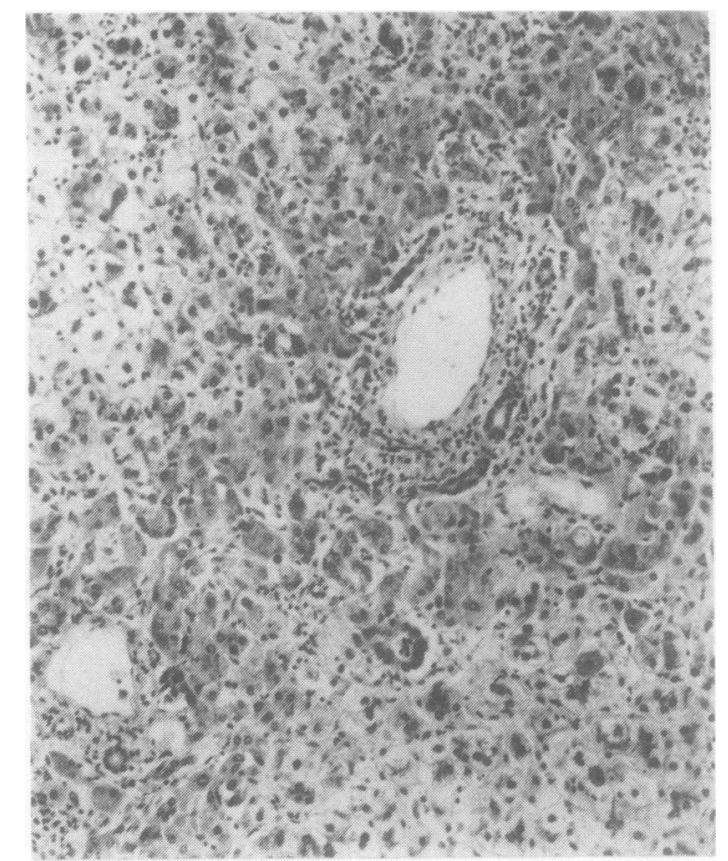

Fig. 11 Acute fatty liver in pregnancy. Note the normal periportal hepatocytes. Haematoxylin and eosin $\times 125$

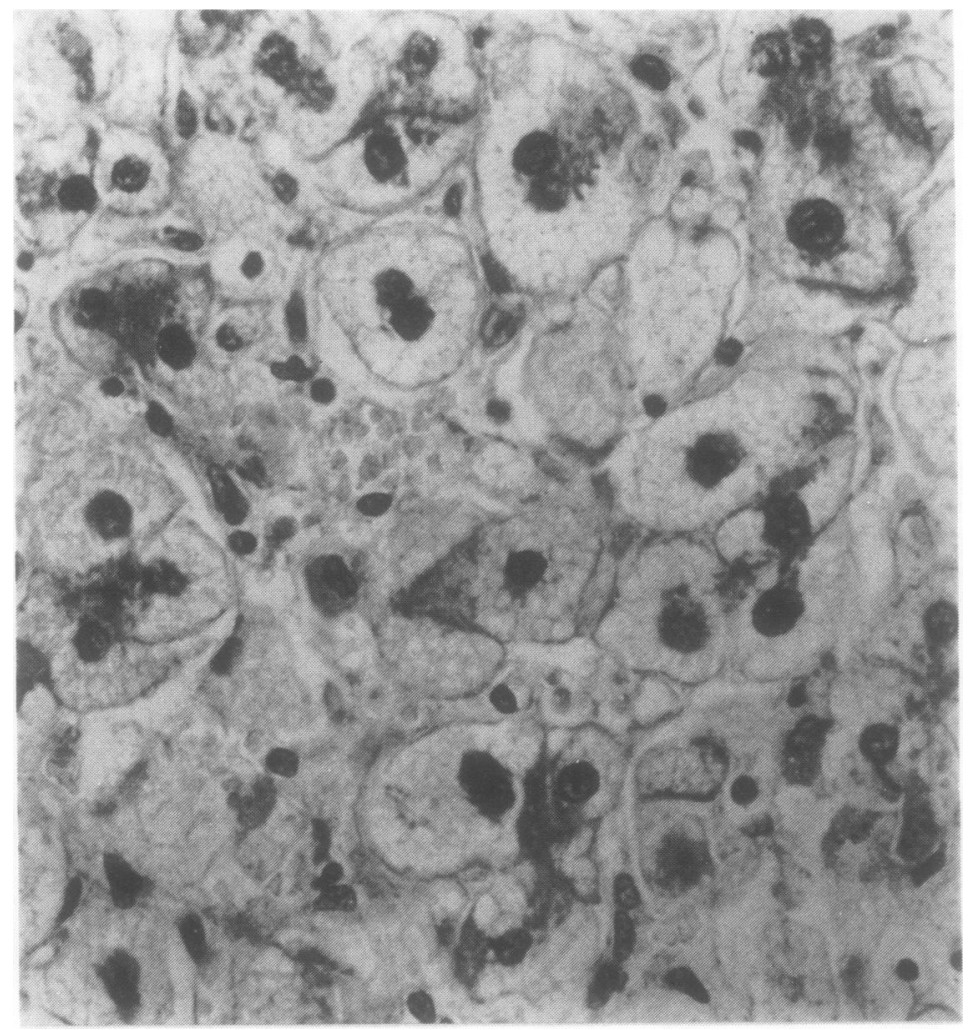

Fig. 12 Acute fatty liver in pregnancy. Note the small vacuoles. Haematoxylin and eosin $\times 490$ 
ing in possible resolution of the effects of the primary event prior to death. The most important anaesthetic complications are aspiration of gastric contents and failure of intubation. On rare occasions equipment failure may be responsible. A few patients may have had intercurrent disease undetected at the time of an emergency anaesthetic or may have failed to reveal facts in their medical histories pertinent to the death. Since it may be argued that all anaesthetic deaths are avoidable it is of paramount importance that all alternative explanations are excluded. In those cases where an adequate reason for the death is not demonstrated, the pathologist should make this clear in his or her report since unwarranted conclusions may result in erroneous assessment of the avoidability or otherwise of the death. Experience would suggest that there should be a section in the Confidential Enquiry on inadequately explained deaths. The failure to explain some of these deaths, must, in the presence of an inadequate autopsy report, be partly blamed on the pathologist concerned. The inclusion of pathological assessments on future reports should allow attention to be drawn to such inadequacies, while the taking of adequate material for histology should greatly reduce their incidence.

Our warm thanks are due to all the Regional Assessors in Pathology who read the draft paper and proffered helpful comments and to our secretaries Mrs O Brooke and Mrs Joy Nice who typed the manuscript.

\section{References}

' Report on Confidential Enquiries into Maternal Deaths in England and Wales 1973-1975. London: HMSO, 1979.

${ }^{2}$ Elliot HR, Abdulla U, Hayes PJ. Pulmonary oedema associated with ritodrine infusion and betamethasone administration in premature labour. $\mathrm{Br}$ Med J 1978;ii:799-800.

${ }^{3}$ Spencer SA, Mann NP, Smith ML, Woolfson AMJ, Benson S. The effects of intravenous therapy during labour on maternal and cord serum sodium levels. Br J Obstet Gynaecol 1981;88:480-3.

${ }^{4}$ Swift PGF, Werthy E. Emery J. Biochemical state of the vitreous humour of infants at necropsy. Arch Dis Child 1974;49:680-5.

${ }^{5}$ Anonymous. Peripartum cardiac failure. Br Med J 1976;i:302-3.

- Sellers WFS, Long DR. Bronchospasm following ergometrine. Anaesthesia 1979;34:909.

${ }^{7}$ Berry CL. The examination of embryonic and fetal material in diagnostic histopathology laboratories. J Clin Pathol 1980;33:317-26.

${ }^{8}$ Langley FA. The perinatal post mortem examination. $J$ Clin Pathol 1971;24:159-69.

" Rushton DI. Examination of products of conception from human pregnancies. J Clin Pathol 1981;34:819-35.

${ }^{10}$ Palomino SJ. Dissecting intramural hematoma of left coronary artery in the puerperium. A case report and survey of the literature. Am J Clin Pathol 1969;51:119-25.

" Abramovich DR, Francis W, Helsby CR. Two cases of ruptured aneurysm of splanchnic arteries in pregnancy with comment on the lesser sac syndrome. Journal of Obstetrics and Gynaecology of the British Commonwealth 1969;76:1037-9.

12 Burnett RA, Carfrae DC. Spontaneous rupture of ovarian artery aneurysm in the puerperium. Two cases and a review of the literature. Br J Obstet Gynaecol 1976;83:744-50.

${ }^{13}$ Corrin B, Spencer H. Some aspects of pulmonary pathology. Shock lung. In: Anthony PP, MacSween RNM, eds. Recent advances in histopathology 11. Edinburgh: ChurchillLivingstone, 1981:83.

${ }^{14}$ Wardle EN, Shanson DC, Lucie NP, et al. Septic shock ACP Symposium J Clin Pathol 1980;33:888-96.

is Pontoppidan H, Geffin B, Lowenstein E. Acute respiratory failure in the adult. $N$ Engl $J$ Med 1972;287:690-698, 743-752, 799-806 (one continuous article subdivided between three journal issues).

${ }^{16}$ Davies MH, Wilkinson SP, Hanid MA, et al. Acute liver disease with encephalopathy and renal failure in late pregnancy and the early puerperium: a study of fourteen patients. Journal of Obstetrics and Gynaecology of the British Commonwealth 1980;87:1005-14.

"Fox H, Langley FA, eds. Postgraduate obstetrical and gynaecological pathology. Oxford: Pergamon Press, 1973:494-5.

${ }^{18}$ Howarth AT. Biochemical indices of osteomalacia in pregnant Asian immigrants in Britain. J Clin Pathol 1976;29:981-3.

${ }^{19}$ Smith AM. Phaeochromocytoma and pregnancy. Journal of Obstetrics and Gynaecology of the British Commonwealth 1973;80:848-51.

${ }^{20}$ Fox H. Placenta accreta 1945-1969. Obstet Gynecol Surv 1972;27:475-90.

${ }^{21}$ Elston CW. Gestation tumour of trophoblast. In: Anthony PP, MacSween RNM, eds. Recent advances in histopathology 11 . Edinburgh: Churchill-Livingstone, 1981:149-62.

${ }^{22}$ Attwood HD. Amniotic fluid embolism. In: Sommers PP, ed. Pathology annual. New York: Appleton-Century-Crofts, 1972:145-72.

${ }^{23}$ Steiner PE, Lushbaugh CC. Maternal pulmonary embolism by amniotic fluid as a cause of obstetric shock and unexpected deaths in obstetrics. JAMA 1941;117:1245-54.

24 Putns S, Desa DJ. Application of a modified Attwood's stain to the study of decalcified bone sections. J Clin Pathol 1977;30:900-3.

${ }^{25}$ Timperley WR. Disseminated intravascular coagulation in forensic pathology. Med Sci Law 1978;18:108-16.

${ }^{26}$ Mant MJ, King EG. Severe acute disseminated intravascular coagulation. A reappraisal of its pathophysiology, clinical significance and therapy, based on 47 patients. Am J Med 1979;67:557-63.

${ }^{27}$ Hyde E, Joyce D, Gurewich V, et al. Intravascular coagulation during pregnancy and the puerperium. Journal of Obstetrics and Gynaecology of the British Commonwealth 1973;80:1059-66.

${ }^{28}$ Sheehan HL, Lynch JB. Patholgy of toxaemia of pregnancy. Edinburgh: Churchill-Livingstone, 1973.

${ }^{29}$ Brosens I, Robertson WB, Dixon HG. The physiological response of the vessels of the placental bed to normal pregnancy. J Pathol Bacteriol 1967;93:569-79.

${ }^{30}$ Robertson WB, Brosens I, Dixon HG. The pathological response of the vessels of the placental bed to hypertensive pregnancy. $J$ Pathol Bacteriol 1967;93:581-91.

${ }^{31}$ Sheppard BL, Bonnar J. The ultrastructure of the arterial supply of the human placenta in pregnancy complicated by fetal growth retardation. Br J Obstet Gynaecol 1976;83:948-59.

Requests for reprints to: Professor Ian Dawson, Department of Pathology, University Hospital, Queen's Medical Centre, Nottingham NG7 2UH, England. 